\title{
A multidimensionalidade da vulnerabilidade acadêmica no programa de assistência estudantil da UTFPR
}

\author{
The multidimensionality of academic vulnerability \\ in the UTFPR student assistance program
}

Juliana Palavezzini ${ }^{1}$

\begin{abstract}
Resumo: Este artigo tem como objetivo analisar a multidimensionalidade da vulnerabilidade acadêmica no programa de assistência estudantil da UTFPR, a partir da utilização do índice de vulnerabilidade do acadêmico (IVA-UTFPR) como um instrumento de classificação. Trata-se de um estudo estatístico e documental, de natureza quanti-qualitativa, fundamentado na revisão bibliográfica. Os resultados demonstram que tanto na composição do IVA-UTFPR quanto no ranking de classificação, a renda per capita não é o único indicador com peso percentual.
\end{abstract}

Palavras-chave: Vulnerabilidade acadêmica. Multidimensionalidade. Assistência estudantil. 
Abstract: This article aims to analyze the multidimensionality of academic vulnerability in the UTFPR student assistance program, using the student's vulnerability index (IVA-UTFPR) as a classification instrument. This is a statistical and documentary study, of a quanti-qualitative nature, based on the bibliographic review. The results demonstrate that both in the composition of the VAT-UTFPR and in the ranking ranking, per capita income is not the only indicator with percentage weight.

Keywords: Academic vulnerability. Multidimensionality. Student assistance.

Universidade Tecnológica Federal do Paraná | Departamento de Educação | Dois Vizinho | PR | Brasil. Contato: julianapalavezzini@gmail.com. ORCID: https://orcid.org/0000-0002-5242-8047

- Recebido em: 8 de março de 2020

- Aprovado em: 5 de novembro de 2020

DOI: http://dx.doi.org/10.1590/S1414-40772021000100012

Este é um artigo publicado em acesso aberto sob uma licença Creative Commons https://creativecommons.org/licenses/by-nc/4.0/ 


\section{Introdução}

Na década de 2000, no contexto do processo de expansão da rede federal de ensino superior, foi regulamentado o PNAES (Programa Nacional de Assistência Estudantil ${ }^{1}$ ), pelo decreto $\mathrm{n}^{\circ} 7234 / 10$ (BRASIL, 2010). Os critérios estabelecidos, bem como os recursos efetivamente destinados ao programa, não possibilitam sua universalização, de modo que os processos seletivos se tornam compulsórios.

Conforme o decreto que regulamenta o PNAES, a assistência estudantil (AE) é um conjunto de princípios e diretrizes que orienta a implantação de ações para garantir o acesso, a permanência e a conclusão de curso dos estudantes das IFES (Instituições Federais de Ensino Superior), na perspectiva de inclusão social, formação ampliada, produção de conhecimento, melhoria do desempenho acadêmico e da qualidade de vida, agindo de forma preventiva nas situações de repetência e evasão, decorrentes da insuficiência de condições financeiras. Os programas de AE desenvolvidos pelas IFES têm se revelado mecanismos imprescindíveis para a permanência e conclusão da maior parte dos estudantes. Dados do FONAPRACE (2019) ${ }^{2}$ (Fórum Nacional de Pró-Reitores de Assuntos Comunitários e Estudantis) revelam que, nos últimos anos, as IFES têm refletido a composição social da população brasileira e, para fazer jus ao atual quadro do perfil discente e oferecer continuidade à tendência de expansão das universidades federais brasileiras, é fundamental a institucionalização e ampliação das políticas de AE.

A tendência de fixar a renda, como único critério de seleção para os programas de AE, vem sendo intensamente discutida e relativizada pelos profissionais da área, seja em âmbito institucional (setores específicos, setores de auditoria, órgãos de gestão), tais como em congressos, nos grupos de trabalhados e também no espaço acadêmico. Atualmente, das 68 universidades federais existentes, aproximadamente 60 têm observado o critério de renda prescrito no PNAES (1,5 salário mínimo per capita), no processo de seleção, contudo, elas têm utilizado, para além da renda, outros critérios (como transporte, habitação e condições de saúde), no processo de classificação dos estudantes.

Na UTFPR, como na maioria das universidades federais, conforme a realidade de cada instituição, os programas de $\mathrm{AE}$ têm observado o critério de renda prescrito no PNAES, contudo, têm sido utilizados, para além da renda, outros critérios (condições de habitação,

\footnotetext{
${ }^{1}$ Inicialmente regulamentado pela portaria normativa no MEC n ${ }^{\circ} 39$ de 12/12/2007.

${ }^{2}$ Para maiores informações, ver: http://www.andifes.org.br/wp-content/uploads/2019/05/V-Pesquisa-Nacionalde-Perfil-Socioeconômico-e-Cultural-dos-as-Graduandos-as-das-IFES-2018.pdf. Acesso em: 19. dez. 2019.
} 
transporte, saúde, etc.), no processo de classificação dos estudantes. Esses novos parâmetros têm sido sistematizados em um índice de vulnerabilidade: IVA-UTFPR.

Nesse contexto, destaca-se o debate sobre vulnerabilidade. As tentativas de mensuração e enfrentamento da vulnerabilidade, em suas diversas dimensões, envolvem questões de natureza teórico-política e técnico-instrumental.

No âmbito teórico-político, conforme estudo que investiga o campo teórico que fundamenta a concepção de vulnerabilidade social, a qual, por sua vez, embasa o tipo de estratégia definida para as políticas sociais, Mauriel (2008), afirma que as transformações recentes das políticas sociais e as reformas nos esquemas de proteção social aparecem como parte de um movimento mais amplo da realidade, situadas a partir da configuração de uma nova ordem econômica global, sob a égide de um padrão de relações internacionais. A autora observa a relação entre as políticas sociais centradas no combate à pobreza e as decisões estratégicas que vêm sendo empreendidas pelos Estados, considerados os principais atores das negociações que se relacionam às políticas sociais. Os processos econômicos e políticos conduzem a paradigmas ou formas individualistas de pensar o social, provocando implicações para as políticas sociais, com uma visão focalizada do indivíduo, não considerando criticamente o contexto socioeconômico, tornando as políticas sociais, mais focalizadas e seletivas. As políticas sociais, sob essa perspectiva, são concebidas como instrumentos para auxiliar na construção de habilidades dos indivíduos e "inseri-los" no modelo de sociabilidade vigente.

No âmbito técnico instrumental, é, especialmente, por intermédio dos indicadores sociais que se busca interpretar (considerando determinados limites) as expressões da vulnerabilidade social. A utilização de indicadores sociais, em qualquer área ou política pública, pressupõe a sua definição conceitual, a delimitação da taxonomia (sistemas classificatórios), bem como suas propriedades.

Uma medida em geral quantitativa dotada de significado social substantivo, usado para substituir, quantificar ou operacionalizar um conceito social abstrato, de interesse teórico [para pesquisa acadêmica] ou programático [para formulação de políticas]. (JANNUZZI, 2002, p. 15).

Para o autor, os indicadores sociais agem como uma medida, frequentemente, quantitativa, que pode ser usada para explanar e interpretar, a partir de uma forma mais estruturada, um conjunto de fenômenos complexos. Os indicadores sociais transformam conceitos abstratos da realidade em medidas que podem ser coletadas, quantificadas, às vezes, mensuradas e analisadas. Nos processos de seleção e classificação socioeconômicas dos programas de $\mathrm{AE}$, tem-se observado, com mais frequência, a utilização de índices de 
vulnerabilidade. As propostas metodológicas utilizam diversos indicadores, considerando as múltiplas dimensões que perpassam os fenômenos de permanência, evasão, desempenho e conclusão de curso.

Partindo das concepções teóricas sobre o tema, traçamos alguns paralelos para conceituar a vulnerabilidade em âmbito acadêmico ${ }^{3}$. A vulnerabilidade acadêmica ${ }^{4}$ não se reduz à insuficiência de renda, pressupondo um caráter e elementos multidimensionais, além de evidenciar as limitações em relação ao acesso, permanência e conclusão desse nível educacional; ademais, pressupõe a interação de fatores de ordem estrutural, objetivos e subjetivos. Esse fenômeno baseia-se na inter-relação de diversos fatores: econômicos, sociais, de saúde, acadêmicos, entre outros. Nesse sentido, os desafios postos não são restritos ao campo conceitual, mas também às práticas institucionais.

Considerando que a AE, na realidade brasileira, se configura como condição essencial para que uma parcela considerável da população possa permanecer e concluir o ensino superior, é essencial que os programas e ações possam intervir positivamente nas principais dificuldades enfrentadas pelos estudantes, mobilizando recursos em diversas áreas, podendo proporcionar condições objetivas para mitigar ou superar os impactos negativos dessas dificuldades. Corroboramos a assertiva de Carvalho (2011, p. 102):

Os fatores que determinam o nível de escolarização dos indivíduos abrangem características pessoais circunstanciais (tais como gênero e cor), características pessoais inatas (esforço, determinação, força de vontade), características familiares (escolaridade e renda dos pais) e características regionais (oferta de ensino na região de moradia).

Nesse sentido, cabe ressaltar que os fatores que influenciam o processo de escolarização são de diversas ordens e vão desde condições estruturais às questões individuais. Dentre as principais dificuldades enfrentadas pelos estudantes, destacamos: dificuldades financeiras, oferta desproporcional (relação demanda/oferta) de vagas/cursos/IES, identificação com o curso/instituição, dificuldades ocasionadas pela carga excessiva de trabalhos estudantis, dificuldades na relação professor-estudante, dificuldades de aprendizado, violências (física, psicológica ou sexual), discriminação, preconceitos, entre outros fatores, que podem impactar

3 Este artigo é um recorte de uma pesquisa de doutoramento cujo objetivo principal foi analisar a multidimensionalidade da vulnerabilidade acadêmica no programa de assistência estudantil da UTFPR, a partir da aplicabilidade do índice de vulnerabilidade do acadêmico, como instrumento de classificação do referido programa.

4 Situando o debate da vulnerabilidade educacional no ensino superior, chamaremos, nesta pesquisa, de vulnerabilidade acadêmica, por estar condicionada às características das instituições de ensino superior. 
a vida acadêmica. Isto posto, assevera-se o caráter multidimensional dos fenômenos de permanência, desempenho e evasão acadêmica.

Evidenciar a perspectiva multidimensional e multifatorial ${ }^{5}$ da permanência dos estudantes em todos os níveis educacionais, especialmente, no ensino superior, é indispensável para evitar simplificações e reduzir a problemática apenas à condição de renda. Apesar das questões de ordem econômica serem peremptórias para permanência dos estudantes no ensino superior, este trabalho compreende e demonstra que há uma pluridimensionalidade de fatores, os quais influenciam, indireta e diretamente, a vida acadêmica. Cabe ressaltar que não se trata de desconsiderar a relevância e até magnitude das condições financeiras à promoção do acesso e permanência no ensino superior, mas de considerar que múltiplos fatores podem interferir, direta ou indiretamente, e com intensidades distintas na vida acadêmica.

\section{A aplicabilidade do IVA-UTFPR}

A partir de 2015, na UTFPR ${ }^{6}$, o programa de assistência ao estudante (PAE) passou a considerar, além do critério de renda per capita, o perfil de vulnerabilidade do acadêmico. $\mathrm{O}$ IVA-UTFPR $^{7}$ busca estabelecer um processo de classificação que contemple as principais causas/motivos das dificuldades de permanência, contribuindo para a melhoria do desempenho acadêmico, além de agir, preventivamente, nas situações de retenção e evasão decorrentes, não somente da insuficiência de condições financeiras, mas também considerando outros fenômenos e desafios que perpassam o cotidiano dos estudantes e seus familiares. A proposta metodológica consiste em uma medida indireta e sintética de identificar e aferir a vulnerabilidade acadêmica, agindo como um critério classificatório ${ }^{8}$ no ranking final de classificação geral.

\footnotetext{
${ }^{5}$ Entendemos que multidimensional são os fenômenos relativos às dimensões da vida humana (econômica, social, cultural etc.) e multifatorial os fenômenos relativos aos fatores institucionais, familiares e individuais. Essa distinção é puramente para efeitos didáticos, pois não há como observar as questões individuais sem considerar as questões econômicas e culturais e assim por diante.

${ }^{6}$ Instituição de ensino superior no formato multi campus, com vocação para a área tecnológica. Ver mais em: www.utfpr.edu.br

${ }^{7}$ O IVA-UTFPR é resultado das decisões de gestão e, sobretudo, da contribuição dos profissionais técnicos administrativos (especialmente, os assistentes sociais). Em 2015, por intermédio de uma portaria do reitor, foi nomeada uma comissão com servidores representantes de todos os treze campi da instituição, com vistas a rever os critérios de seleção e classificação do programa de assistência ao estudante (PAE) da UTFPR.

${ }^{8} \mathrm{Na}$ execução do programa, o processo de classificação dos estudantes é realizado conforme pontuação obtida com o IVA-UTFPR, considerando uma ordem decrescente (quanto maior a pontuação, maior a vulnerabilidade), a partir dos dados dos treze campi, sistematizados em uma lista única.
} 
As variáveis estudadas foram os indicadores que compõem o IVA-UTFPR, conforme constam nos respectivos editais dos anos estudados, quais sejam: renda familiar per capita; ser ou não cotista; receber outras bolsas; condição de residência do aluno; número de integrantes da família; ter outro familiar cursando graduação; ser a primeira graduação do estudante; condição de permanência na residência (família); condição de saúde do estudante; condição de saúde na família do estudante; situações atípicas do estudante e/ou família. No IVA-UTFPR, a unidade de medida (pontuação) é escalonada a partir de valores extremos normativos (piso e teto), sendo que: quanto mais alta a pontuação, maior será o índice calculado e, portanto, maior sua vulnerabilidade. Quanto mais baixa a pontuação, menor será o índice calculado e, portanto, menor sua vulnerabilidade.

O recorte temporal definido para análise dos dados compreendeu os anos de 2017, 2018 e 2019, por apresentarem maiores semelhanças, por serem processos anuais e pela atualidade dos dados.

Para analisar a aplicabilidade do IVA-UTFPR, foi realizado um estudo estatístico, o qual, entre outros dados, investigou a correlação entre os indicadores utilizados na composição do IVA-UTFPR e identificou o peso dos indicadores, tanto na composição do IVA-UTFPR quanto no ranking de classificação geral. O estudo também investigou o desempenho do indicador de renda per capita na composição do IVA-UTFPR e o ranking final de classificação geral.

O instrumento de medida utilizado - o banco de dados (BD) - resultou das planilhas de Excel, que sintetizam informações do questionário socioeconômico, utilizado na avaliação socioeconômica. O lançamento dos dados foi realizado por meio do Microsoft Excel, enquanto que a análise foi realizada a partir do software Statística, versão 13. Como procedimento metodológico, o tratamento estatístico calculou a fidedignidade e validade da pesquisa, com base no Alfa de Cronbach. A pesquisa considera um erro amostral igual a 5\% e margem de confiança de 95\%. O quadro, a seguir, sintetiza dados da população geral e a amostragem utilizada nos três anos estudados.

Quadro 1 - Dados gerais: população e amostragem

\begin{tabular}{|c|c|c|c|}
\hline Ano de Referência & $\mathbf{N}^{\circ}$ contemplados & Amostragem Mínima & Estudantes avaliados \\
\hline $\mathbf{2 0 1 7}$ & 2.873 & 779 & $\mathbf{2 2 4 1}$ \\
\hline $\mathbf{2 0 1 8}$ & 4.474 & 862 & $\mathbf{3 8 1 8}$ \\
\hline $\mathbf{2 0 1 9}$ & 4.639 & 880 & $\mathbf{4 . 6 3 9}$ \\
\hline
\end{tabular}

Fonte: Dados auxílio Estudantil UTFPR 2017/2018/2019. Sistematização própria. 
O quadro 2 sintetiza os dados iniciais sobre a variável renda e faixas de pontuação, tanto do IVA-UTFPR (do estudante) quanto do ranking final (listagem com todos os contemplados). Esses dados iniciais são complementados com os demais estudos que envolvem a variável renda no decorrer da pesquisa, referente aos anos de 2017 a 2019.

Quadro 2 - Dados gerais renda e pontuação da renda no IVA-UTFPR

\begin{tabular}{|c|c|c|c|c|}
\hline \multicolumn{5}{|c|}{ QUADRO REFERENTE AO ANO DE 2017} \\
\hline VARIÁVEL & N. AMOSTRA & $\begin{array}{l}\text { VALOR } \\
\text { MÉDIO }\end{array}$ & $\begin{array}{c}\text { VALOR } \\
\text { MÍNIMO }\end{array}$ & $\begin{array}{c}\text { VALOR } \\
\text { MÁXIMO }\end{array}$ \\
\hline Renda & 2241 & $\mathrm{R} \$ 1423,7$ & 0 & $\mathrm{R} \$ 6391,1$ \\
\hline Renda per capita & 2241 & $\mathrm{R} \$ 398,5$ & 0 & $\mathrm{R} \$ 1231,7$ \\
\hline Pontuação renda & 2241 & 72,1 & 15 & 90 \\
\hline IVA-UTFPR & & 141,1 & 125 & 205 \\
\hline \multicolumn{5}{|c|}{ QUADRO REFERENTE AO ANO DE 2018} \\
\hline & N. AMOSTRA & $\begin{array}{l}\text { VALOR } \\
\text { MÉDIO }\end{array}$ & $\begin{array}{l}\text { VALOR } \\
\text { MÍNIMO }\end{array}$ & $\begin{array}{c}\text { VALOR } \\
\text { MÁXIMO }\end{array}$ \\
\hline Renda & 3818 & $\mathrm{R} \$ 1904,39$ & 0 & $\mathrm{R} \$ 8600$ \\
\hline Renda per capita & 3818 & $\mathrm{R} \$ 568,01$ & 0 & $\mathrm{R} \$ 1431,00$ \\
\hline Pontuação renda & 3818 & 61,05 & 15 & 90 \\
\hline IVA-UTFPR & 3818 & 123,73 & 45 & 215 \\
\hline \multicolumn{5}{|c|}{ QUADRO REFERENTE AO ANO DE 2019} \\
\hline & N. AMOSTRA & $\begin{array}{l}\text { VALOR } \\
\text { MÉDIO } \\
\end{array}$ & $\begin{array}{c}\text { VALOR } \\
\text { MÍNIMO }\end{array}$ & $\begin{array}{c}\text { VALOR } \\
\text { MÁXIMO }\end{array}$ \\
\hline Renda & 4639 & $\mathrm{R} \$ 1761,71$ & 0 & $\mathrm{R} \$ 8134,32$ \\
\hline Renda per capita & 4639 & $\mathrm{R} \$ 619,70$ & 0 & $\mathrm{R} \$ 1473,32$ \\
\hline Pontuação renda & 4639 & 60 & 10 & 100 \\
\hline IVA-UTFPR & 4639 & 85 & 15 & 180 \\
\hline
\end{tabular}

Fonte: Dados auxílio Estudantil UTFPR 2017/2018/2019. Sistematização própria.

Os dados indicam em quais anos/faixas houve maior concentração de contemplados. Em 2017, a média da pontuação do IVA-UTFPR foi de 141 pontos; a pontuação mínima foi de 125 pontos e a máxima, 205 pontos. Em 2018, a média da pontuação do IVA-UTFPR foi de 124 pontos, sendo que o valor mínimo foi de 45 pontos e o máximo de 215 pontos. A pontuação média, obtida no ranking de classificação do IVA-UTFPR, em 2019, foi de 85 pontos, sendo 15 a pontuação mínima e 180 a pontuação máxima.

É possível afirmar que houve aumento gradual tanto no valor médio da renda total (bruta) quanto da renda per capita, nos anos pesquisados, sendo que o maior aumento foi verificado entre 2017/2018/2019, conforme evidenciado no quadro dois. Considerando a média de pontuação no ranking de classificação final do IVA-UTFPR, entre os anos estudados, observa-se uma queda na pontuação total, bem como na pontuação atribuída pela renda per capita. Cabe ressaltar que esse dado não indica, necessariamente, uma diminuição da vulnerabilidade dos acadêmicos, tendo em vista que os indicadores e a pontuação atribuída 
sofreram alterações, sendo que a média de pontuação do índice e do ranking foi impactada por essas alterações.

Em seguida, identificamos as variáveis com maior peso na composição do índice e também do ranking. A sistematização dos dados permitiu identificar as variáveis com maior peso percentual, na composição do IVA-UTFPR, e também do Ranking, no ano de 2017:

Tabela 1 - Peso das variáveis em relação IVA-UTFPR X Ranking 2017

\begin{tabular}{crr}
\hline Variáveis/indicadores IVA-UTFPR - 2017 & $\mathrm{r}^{2}$-\% IVA-UTFPR & $\mathrm{r}^{2}$-\% Rank. \\
\hline Pontuação Renda per capita & 20,7 & 31,2 \\
Pontuação Situações atípicas & 5,4 & 2,1 \\
Condição de Moradia do Estudante & 4,2 & 8,5 \\
Reside com & 3,5 & 2,4 \\
\hline
\end{tabular}

Fonte: Dados auxílio Estudantil UTFPR 2017, sistematização própria.

Onde: $\mathrm{O}^{2}$ significa que houve uma associação entre as variáveis, ou seja, uma influencia na outra.

Quanto maior o valor de $\mathrm{r}^{2}$ maior é a associação entre as variáveis.

A pontuação da renda, em 2017, teve o maior peso percentual, 20,7\%, na composição do IVA-UTFPR. Já em relação ao ranking, a pontuação da renda per capita teve um peso percentual de $31.2 \%$, sendo o indicador mais expressivo. A tabela, a seguir, sintetiza os dados referentes às variáveis com maior peso, em relação ao IVA-UTFPR e em relação ao Ranking, para o ano de 2018.

Tabela 2 - Peso das variáveis em relação IVA-UTFPR X Ranking 2018

\begin{tabular}{ccc}
\hline Variáveis/indicadores IVA-UTFPR - 2018 & r $^{2}$ \% IVA-UTFPR & r $^{2}$-\% Rank. \\
\hline Pontuação Renda per capita & 57 & 59,7 \\
Pontuação Situações atípicas & 5,7 & 5,1 \\
Condição de Moradia-Estudante & 3,7 & 3 \\
Reside com & 3,1 & 2,4 \\
\hline
\end{tabular}

Fonte: Dados auxílio Estudantil UTFPR 2018, sistematização própria.

Onde: $\mathrm{O}^{2}$ significa que houve uma associação entre as variáveis, ou seja, uma influencia na outra. Quanto maior o valor de $\mathrm{r}^{2}$ maior é a associação entre as variáveis.

Em 2018, apesar de não sofrer alteração no valor (não houve alteração no critério de avaliação, nem na pontuação atribuída ao indicador), o peso da pontuação da renda familiar per capita foi maior em relação ao ano de 2017; o peso dessa variável foi de 57\%. Já em relação ao ranking, a pontuação da renda per capita teve um peso final de 59,7\%, sendo o indicador mais expressivo. A seguir, sintetizamos os dados referentes às variáveis com maior peso em relação ao IVA-UTFPR e em relação ao Ranking, para o ano de 2019. 
Tabela 3 - Peso das variáveis em relação IVA-UTFPR X Ranking 2019

\begin{tabular}{crc}
\hline Variáveis/indicadores IVA-UTFPR - 2019 & r $^{2}$ \% IVA-UTFPR & r $^{2}$-\% Rank. \\
\hline Situação moradia estudante & 2,76 & 2,56 \\
Situação moradia família & 3,28 & 2,76 \\
Doença estudante & 0,55 & 0,38 \\
Doença família & 0,21 & 12,25 \\
Pont. Forma de deslocamento & 3,53 & 22,09 \\
Situação atípica & 3,53 & 1,93 \\
Pontuação renda & 78,68 & 80,46 \\
Cota & 0,51 & 0,3 \\
\hline
\end{tabular}

Fonte: Dados auxílio Estudantil UTFPR 2017/2019, sistematização própria.

Onde: $\mathrm{O} \mathrm{r}^{2}$ significa que houve uma associação entre as variáveis, ou seja, uma influencia na outra. Quanto maior o valor de $r^{2}$ maior é a associação entre as variáveis.

Considerando os dados dos três anos pesquisados, observa-se que a pontuação, obtida a partir da renda per capita, continua compondo o indicador com maior peso percentual, o que mais impacta na determinação do IVA-UTFPR, passando de 20,7\%, em 2017, para 57\%, em 2018, chegando a 78,68\%, em 2019. Em relação ao Ranking, o peso percentual da renda per capita também se sobrepõe aos outros indicadores, passando de 31,2\%, em 2017, para 9,7\%, em 2018, chegando a 80,46\%, em 2019.

Atualmente, o indicador renda per capita é o que possui maior peso definindo, tanto em relação à pontuação do IVA-UTFPR como ao ranking de classificação, ou seja, é um instrumento que continua priorizando o acadêmico mais vulnerável em termos econômicos. Apesar da relevância do indicador de renda não ser o único, outros indicadores, como 'situações atípicas', 'condições de moradia' e 'forma de deslocamento', são importantes estatísticas na composição do IVA-UTFPR, reforçando a compreensão de que a renda per capita não é o único indicador de vulnerabilidade dos estudantes.

Os dados, a seguir, demonstram a relação de causa-efeito entre as variáveis, primeiro, em relação ao índice e, em seguida, em relação ao ranking. Essa tabela mostra a evolução do comportamento das variáveis que compõem o IVA-UTFPR. Os dados mostram a alteração no comportamento das variáveis (indicadores) e pesos que compõem o IVA-UTFPR. Em relação ao IVA-UTFPR, o estudo está demonstrado na tabela, a seguir: 
Tabela 4 - Relação causa/efeito (peso) das variáveis em relação ao IVA-UTFPR 2017/2018/2019

\begin{tabular}{llccc}
\hline \multicolumn{1}{c}{ Indicador/Variável } & $\begin{array}{c}\mathbf{r}^{2} \text {-IVA-UTFPR } \\
\mathbf{2 0 1 7}\end{array}$ & $\begin{array}{c}\mathbf{r}^{\mathbf{2}} \text {-IVA-UTFPR } \\
\mathbf{2 0 1 8}\end{array}$ & $\begin{array}{c}\mathbf{r}^{\mathbf{2}} \text {-IVA- } \\
\text { UTFPR 2019 }\end{array}$ \\
\hline 1. Integrantes & $0,0 *$ & 1,8 & --- \\
2. Cota & 1,1 & 1,2 & 0,51 \\
3. Bolsas & 0,0 & 0,1 & --- \\
4. Residência (reside com) & $\mathbf{3 , 5}$ & $\mathbf{3 , 7}$ & $\mathbf{2 , 7 6}$ \\
5. Fam. Cursando Graduação & 1,2 & 0,4 & 0,0 \\
6. Outro Curso Graduação** & 0,5 & 0,7 & --- \\
7. Condição de residência Família & $\mathbf{2 , 4}$ & $\mathbf{5 , 7}$ & $\mathbf{3 , 2 8}$ \\
8. Doença & 0,1 & 0,2 & 0,55 \\
9. Doença na Família & 1,2 & 0,5 & 0,21 \\
10. Forma Deslocamento & 0,8 & 1,3 & 0,19 \\
11. Situação Atípica & $\mathbf{5 , 4}$ & $\mathbf{3 , 1}$ & $\mathbf{3 , 5 3}$ \\
12. Pontuação Renda Per Capita & $\mathbf{2 0 , 7}$ & $\mathbf{5 7 , 0}$ & $\mathbf{7 8 , 6 8}$ \\
Moradia do Estudante*** & $\mathbf{4 , 2}$ & $\mathbf{- - -}$ & $\mathbf{- - - - -}$ \\
\hline
\end{tabular}

Fonte: Dados auxílio Estudantil UTFPR 2017/2018/2019, sistematização própria.

*Valores menores do que 2,00 são pouco relevantes. ** Retirado em 2019, ***só existiu em 2017.

Os indicadores com maior peso, no ano de 2017, foram, respectivamente: renda per capita, situação atípica, condições de moradia do estudante, residência do estudante (com quem reside) e condição de permanência na residência da família. Em 2018, os indicadores com maior peso foram, respectivamente: renda per capita, condição de permanência na residência da família, residência do estudante (com quem reside) e situação atípica. Em 2019, os indicadores com maior peso foram, respectivamente: renda per capita, situações atípicas na família, condição de residência da família e residência do estudante (com quem reside). Nos três últimos anos, vemos que, além da renda per capita, outras variáveis são importantes à composição do IVA-UTFPR, tais como: "reside com", condição de residência da família e situações atípicas.

$\mathrm{Na}$ tabela a seguir, é possível observar a relação de causa e efeito das variáveis (indicadores), entretanto, agora, em relação ao ranking de classificação.

Tabela 5 - Relação causa/efeito (peso) das variáveis em relação ao ranking 2017/2018/2019.

\begin{tabular}{|c|c|c|c|}
\hline Indicador/variável & Ranking 2017 & Ranking 2018 & Ranking 2019 \\
\hline 1. Integrantes & $0,1^{*}$ & 1,0 & ---- \\
\hline 2. Cota & 0,8 & 0,9 & 0,00 \\
\hline 3. Bolsas & 0,2 & 0,0 & 0,00 \\
\hline 4. Residência (reside com) & 2,4 & $3, \mathbf{0}$ & 2,56 \\
\hline 5. Fam. Cursando Graduação & 0,7 & 0,3 & 0,00 \\
\hline 6. Outro Curso Graduação & 0,4 & 0,5 & ---- \\
\hline 7. Condição de residência Família & 1,4 & 5,1 & 2,76 \\
\hline 8. Doença & 0,1 & 0,2 & 0,38 \\
\hline 9. Doença na Família & 0,5 & 0,3 & 0,12 \\
\hline 10. Forma Deslocamento & 0,6 & 1,2 & 0,20 \\
\hline 11. Situação Atípica & 2,1 & 2,4 & 1,93 \\
\hline 12. Pontuação Renda Per Capita & 31,2 & 59,7 & 80,46 \\
\hline 13. Moradia do estudante* & 2,6 & ---- & ---- \\
\hline
\end{tabular}

Fonte: Dados auxílio Estudantil UTFPR 2017/2018/2019, sistematização própria. 
*Valores menores do que 2,0 são pouco relevantes. ** Retirado em 2019, ***só existiu em 2017.

A tabela revela que, nos três anos pesquisados, as variáveis "renda per capita", "reside com" e "situação atípica", também são as que mais influenciaram no ranking. Resultado similar foi encontrado na tabela anterior sobre a influência no IVA-UTFPR. Observa-se que, nos três anos, houve outras variáveis com peso na composição do índice, reforçando que a renda não é a única variável, embora tenha um peso significativo. Assim como entre 2017 e 2018, houve alterações das variáveis/indicadores (situação de moradia do estudante deixou de existir); a variável "condições de permanência na residência da família" tornou-se mais forte, em 2018. Interessante assinalar que a pontuação obtida, a partir da pontuação da renda per capita, tornouse mais forte ainda depois da estratificação em 10 categorias. Em 2019, o peso da renda per capita correspondeu a $78 \%$ do IVA e peso de $81 \%$ no ranking.

Observa-se que os indicadores: cotista, outras bolsas, outro familiar cursando graduação, outro curso de graduação, doença (do estudante e da família), forma de deslocamento, não têm significância estatística, por apresentarem baixa frequência e/ou por atribuírem pontuação à maioria expressiva dos acadêmicos. Nota-se que, em relação ao indicador "cotista", nos dois anos avaliados, não há significado estatístico. Referente a esse dado, cabe uma ponderação: se, estatisticamente, o indicador cotista não apresenta relevância (porque, como a maioria dos estudantes são cotistas, então, a maioria recebe os 10 pontos), contudo, ao observar analítica e criticamente o dado, entende-se como um indicador relevante, pois demostra que a maioria dos contemplados no programa são cotistas, ou seja, provenientes de segmentos sociais vulnerabilizados historicamente. Esse dado nos permite aventar que há uma simbiose entre as políticas de assistência estudantil e de cotas, fato que requer análises mais aprofundadas e consistentes, o que ultrapassa os limites deste artigo.

O mesmo acontece com o indicador "receber outras bolsas". Como a maioria dos estudantes não recebem outra modalidade de bolsa, a maioria "recebe" pontuação nesse indicador, fazendo com que ele perca a validade estatística. No ano de 2018, em termos percentuais, temos $13,75 \%$ (525) dos contemplados que recebiam outra modalidade de bolsa e 86,24\% (3293) não contemplados. Pelo estudo de correlação, o indicador "outro familiar cursando graduação", por apresentar baixa frequência, também não demonstra relevância estatística. Já em relação ao indicador "outro curso de graduação", apesar de também não ser frequente, diferentemente dos outros indicadores, essa variável tem um princípio negativo. Desse modo, ela tem sua importância por não valorizar quem já tem uma graduação. Observando os indicadores "doença estudante e doença na família", nota-se que a frequência 
dos casos de doença na família é maior do que do estudante sozinho, porém, a pontuação atribuída não tem relevância estatística.

O estudo de causa/efeito é importante para definir os indicadores que mais impactam na definição do índice e do ranking. Cabe destacar que, embora alguns indicadores não sejam determinantes à composição do índice, representam questões complexas e relevantes para o diagnóstico específico de alguns casos, além de evidenciar as concepções teóricas e técnicas da equipe que formulou os instrumentos. Algumas dimensões e indicadores possuem baixa validade estatística, devendo ser observados pela sua relevância social, consideradas as concepções da equipe técnica que os elegeram.

O estudo dos indicadores com maior influência da definição, tanto do índice quanto do ranking, permite que a equipe técnica identifique se as pontuações atribuídas estão de acordo com o objetivo do instrumento e se são coerentes com pressupostos teóricos que fundamentam a escolha da sua utilização. Cabe destacar que é o aporte teórico que vai propiciar consistência a um indicador; nele, devem estar explicitadas as variáveis e categorias analíticas relevantes, bem como o encadeamento causal ou lógico que as relaciona. As concepções teóricas e conceituais da equipe técnica motivam e fundamentam a opção pela utilização dos indicadores e seus pesos percentuais. "A validade de um indicador corresponde ao grau de proximidade entre o conceito e a medida, ou seja, sua capacidade de refletir, o conceito abstrato a que o indicador se propõe a "substituir" ou “operacionalizar" (JANNUZZI, 2002, p. 26).

Foi realizado um estudo de correlação (2018/2019), visando identificar a relação entre as variáveis, de maneira a identificar se, quando um indicador varia, o outro varia também. Em resumo, a força de uma relação entre duas variáveis nos propicia o grau com que uma variável tende a variar quando a outra varia ${ }^{9}$. O estudo foi realizado com base na metodologia do Coeficiente de Pearson, por meio da qual identificou-se a força e a direção das variáveis utilizadas. O coeficiente de correlação de Pearson ${ }^{10}$, também chamado de "coeficiente de correlação" ou simplesmente de " $\rho$ de Pearson", mede o grau da correlação (e a direção dessa correlação - se positiva ou negativa) entre duas variáveis de escala métrica. A interpretação do coeficiente de Pearson varia de -1 a 1 . O coeficiente de correlação de Pearson (r) é uma medida de associação linear entre variáveis. Ela é expressa em uma escala, indo de

\footnotetext{
${ }^{9}$ Dito de outro modo, baseado na medida de correlação entre duas variáveis, pode-se saber se o conhecimento de valores de uma das variáveis permite a previsão de valores da outra variável.

${ }^{10}$ A opção pelo procedimento foi em função da normalidade dos dados, observada pelo teste de KolmogorovSmirnov, pelo tamanho amostral e pela característica dos dados, sugerindo estatística paramétrica. O grau de confiabilidade foi estabelecido com um $\mathrm{p} \leq 0,01$.
} 
-1 (correlação negativa perfeita) a +1 (correlação positiva perfeita). Segundo Roque (2009), a variável que mede a força de uma correlação (nessa escala de $-1 \mathrm{a}+1$ ) é chamada de coeficiente de correlação (representado pela letra r). Desse modo, se uma variável tende a aumentar quando a outra aumenta, dizemos que a correlação é positiva. Por outro lado, se uma variável tende a diminuir quando a outra aumenta, dizemos que a correlação é negativa. Já uma correlação igual a zero, indica que uma variação, em uma das variáveis (aumento ou diminuição), não influencia a outra ${ }^{11}$. Portanto, o estudo de correlação buscou identificar quanto um indicador interfere e influencia o outro, ou seja, o estudo foi realizado para medir a relação entre as variáveis; quanto mais próximo de 1 , mais forte a variável:

Tabela 6 - Coeficiente de correlação entre os indicadores do IVA-UTFPR 2018/2019.

\begin{tabular}{|c|c|c|c|}
\hline Indicador/Variável & $\begin{array}{c}\text { IVA-UTFPR } \\
2018 \\
\end{array}$ & N. de Categorias & $\begin{array}{c}\text { IVA-UTFPR } \\
2019 \\
\end{array}$ \\
\hline 1. Bolsas & $0,038342 *$ & 2 & 0,002 \\
\hline 2. Doença do Estudante & 0,041031 & 2 & 0,074 \\
\hline 3. Fam. Cursando Graduação & 0,066820 & 2 & 0,001 \\
\hline 4. Doença na família & 0,067704 & 2 & 0,046 \\
\hline 5. Outro curso de graduação & 0,084179 & 2 & ---- \\
\hline 6. Cotas & 0,108955 & 2 & ------ \\
\hline 7. Forma de Deslocamento & 0,112120 & 3 & 0,060 \\
\hline 8. Número de integrantes $(5-25)$ & 0,132444 & 5 & $\mathbf{0 , 1 7 7}$ \\
\hline 9. Pontuação Situação Atípica (20- 40) & 0,176591 & var. & 0,188 \\
\hline 10. Reside com (5-20) & 0,191905 & 4 e 5 & 0,166 \\
\hline 11. Permanência da Família (0 20) & 0,238405 & 4 & 0,181 \\
\hline 12. Pontuação de Renda (15-90) & 0,755008 & 6 e 10 & $\mathbf{0 , 8 8 7}$ \\
\hline
\end{tabular}

Fonte: Dados auxílio Estudantil UTFPR 2018/2019, sistematização própria. *Quanto mais próximo de 1, mais forte a variável.

O estudo do coeficiente de correlação entre as variáveis/indicadores demostrou que há correlação positiva maior entre as categorias/indicadores: "Número de integrantes na família", "Situações Atípicas", "Reside com", "Permanência da família" e "pontuação de renda". Lembrando que o estudo do coeficiente correlação é o tipo de medida que se usa quando se quer saber se duas variáveis possuem algum tipo de relação, de maneira que, quando uma varia, a outra varia também.

Os dados acima demonstram, ainda, que o coeficiente de relação está relacionado com o número de categorias que compõem cada indicador na formação do IVA-UTFPR, ou seja,

\footnotetext{
${ }^{11}$ No estudo realizado, os dados de correlação permitiram aplicação de técnica linear simples $\left(\mathrm{r}^{2}\right)$ para sugestão de relações de causa-efeito. Segundo Roque (2019), em geral, quando há uma correlação significante entre duas variáveis, A e B, pode haver várias possíveis interpretações para a relação entre elas, tais como: a variável A tem um efeito causal sobre a variável B; a variável B tem um efeito causal sobre a variável A; tanto A como B estão relacionadas a alguma outra variável; apesar de significante a um nível $\alpha$ (por exemplo, 0,05), a correlação entre A e B não é real e o valor do coeficiente de correlação é apenas fruto de uma coincidência.
} 
quanto maior a quantidade de categorias no indicador, maior a sua força na composição do IVA-UTFPR. No caso do estudo em tela, a observação da correlação permite afirmar que: quanto mais vulnerável é o estudante, menor é a sua renda; quanto mais doente é a família do estudante, maior seu IVA; quanto mais bem posicionado o estudante no ranking, maior é a sua vulnerabilidade.

Finalmente, realizou-se um estudo de medidas repetidas, visando identificar o impacto da variável renda na permanência dos estudantes. Os dados complementam os estudos iniciais da variável renda per capita e permitem identificar a evolução dessa variável nos anos pesquisados. A avaliação de medidas repetidas é um procedimento estatístico que permite comparar variáveis e/ou comportamentos contínuos em duas observações distintas. Inicialmente, houve a separação dos estudantes, considerando os que foram contemplados, nos anos 2017/2018, sendo que foram 1.334 estudantes; em seguida, aplicou-se técnica das medidas repetidas, escolhendo a variável renda per capita, por ter a maior força na composição do IVAUTFPR; por fim, analisou-se a idade dos acadêmicos para comprovar o recorte temporal (em que a idade inicial era de 22,35 anos e a idade, no processo final, foi de 23,36 anos). O resultado indica a variação de um ano, apontando que há uma medida repetida de um intervalo de 12 meses.

Figura 1 - Estudo de medidas repetidas: renda per capita 2017 X renda 2018

Box \& Whisker Plot

Renda 2017 vs. Renda 2018

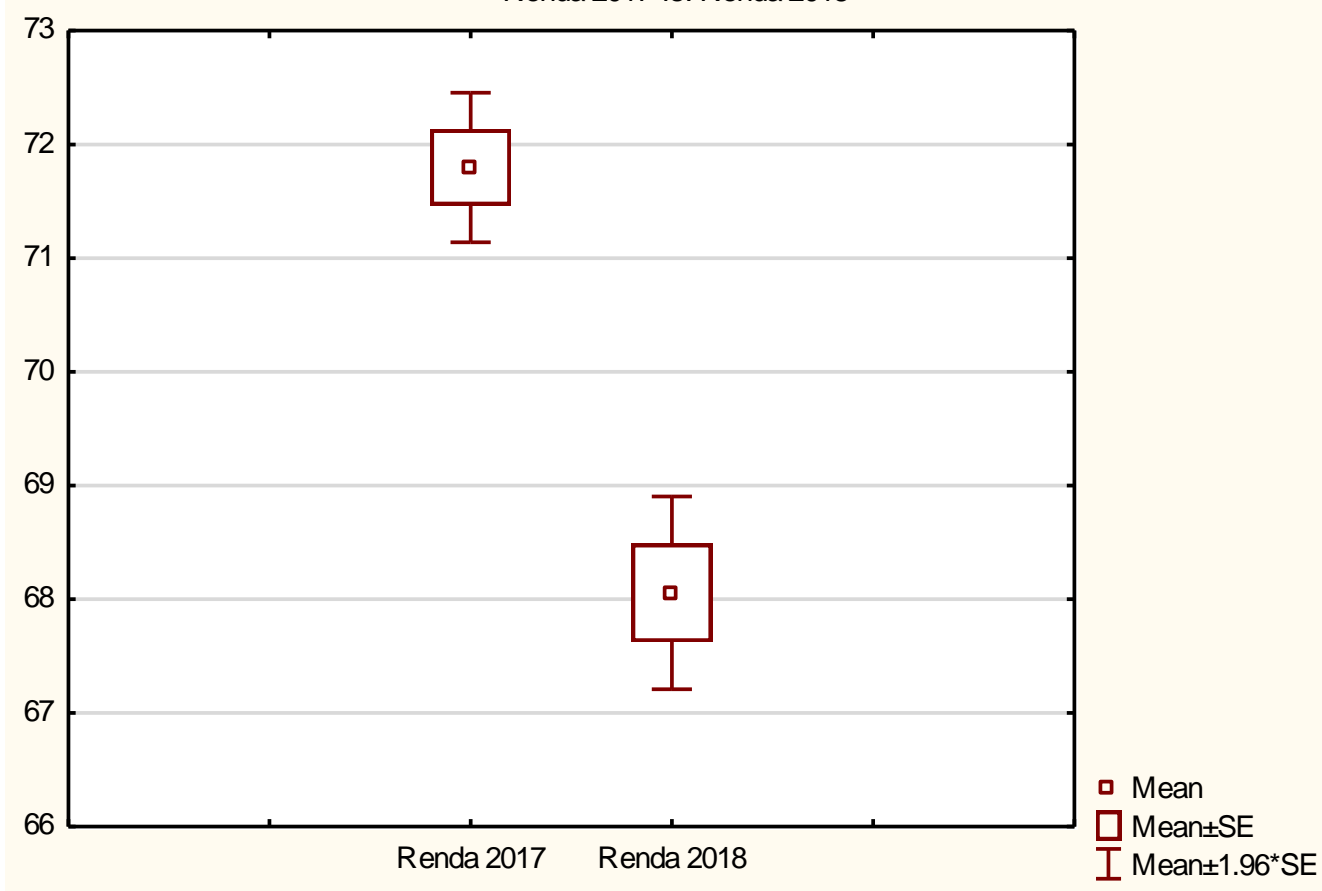

Fonte UTFPR/ASSAE 2017/2018. Sistematização própria. 
Dos 1334 estudantes amostrados (com medidas repetidas), observou-se que, em 2017, fizeram, em média, 72 pontos com o indicador de renda per capita. Em 2018, essa pontuação caiu para 68 pontos. Isso significa que, se houve queda do peso percentual da pontuação da renda per capita, na composição final do IVA-UTFPR, corroboram-se os dados já identificados anteriormente, nos dados descritivos iniciais sobre renda. A queda de pontos revela que houve uma menor pontuação do indicador de renda; em consequência, diminuiu a faixa de pontuação, apontando uma diminuição do IVA-UTFPR.

A figura 2 refere-se ao estudo de medidas repetidas sobre a variável renda bruta e renda per capita, referentes aos três anos estudados, sendo, respectivamente: 1 , referente aos dados de 2017; 2, aos dados de 2018; e 3, aos dados de 2019.

Figura 2 - Estudo de medidas repetidas: renda familiar

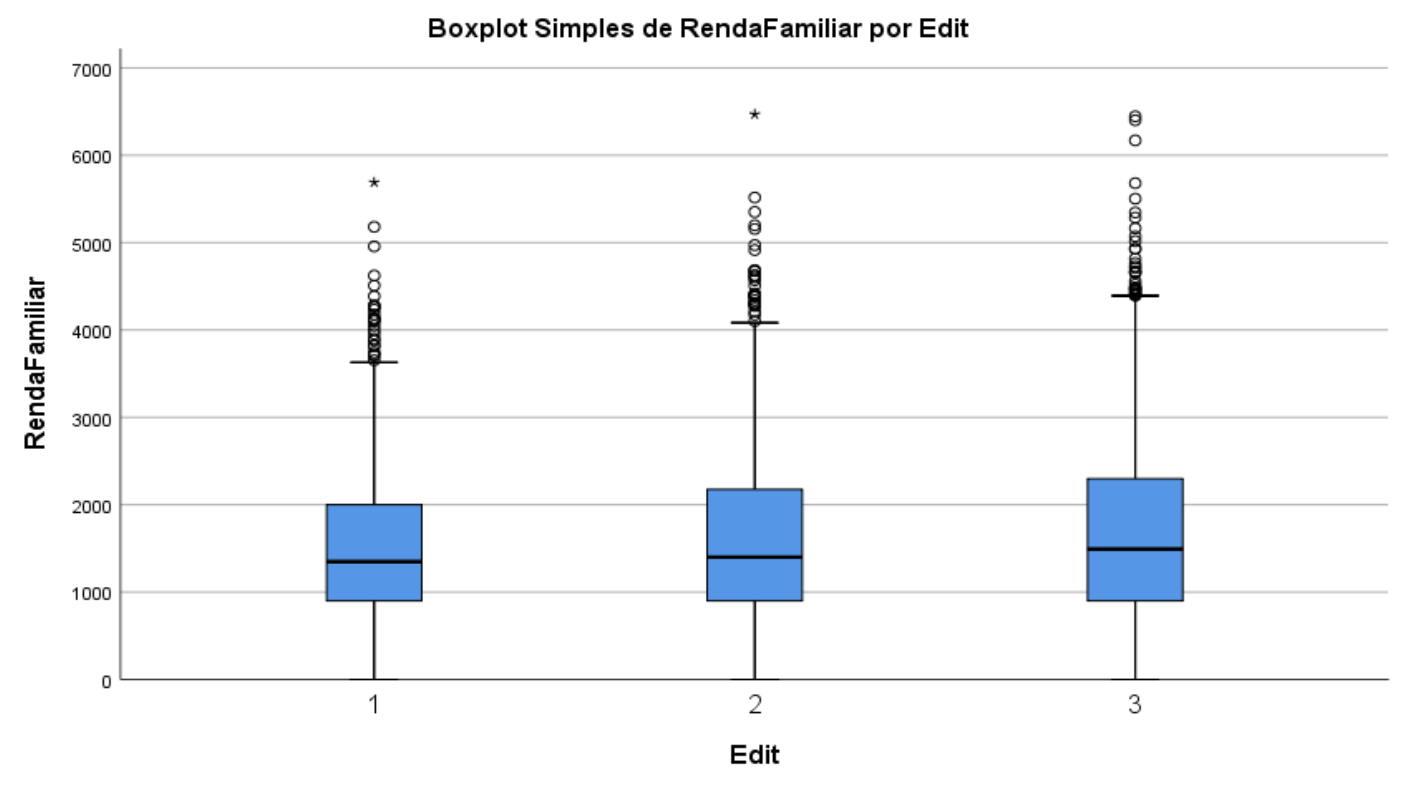

Fonte UTFPR/ASSAE 2017/2018/2019 Sistematização própria.

Foram encontrados 1.963 estudantes com medidas repedidas e o resultado demonstra que a renda familiar dos alunos contemplados vem aumentando no decorrer dos três últimos anos. A renda familiar (bruta) média aumentou progressivamente no decorrer dos três anos pesquisados. Em 2017, a renda familiar média foi de $\mathrm{R} \$ 1.464,28$; em 2018, a renda média foi de $\mathrm{R} \$ 1.576,17$ e, em 2019, a renda familiar média foi de $\mathrm{R} \$ 1.667,93$.

A figura 3, refere-se à renda per capita para os três anos pesquisados. O estudo demonstra que a renda familiar per capita também aumentou, no decorrer dos três anos 
pesquisados, para os estudantes contemplados nesses três anos. Em 2017, a renda per capita foi de $\mathrm{R} \$ 407,42$; em 2018, de R\$ 448,39 e, em 2019, de R\$ 517,52.

Figura 3 - Estudo de medidas repetidas: renda familiar per capita.

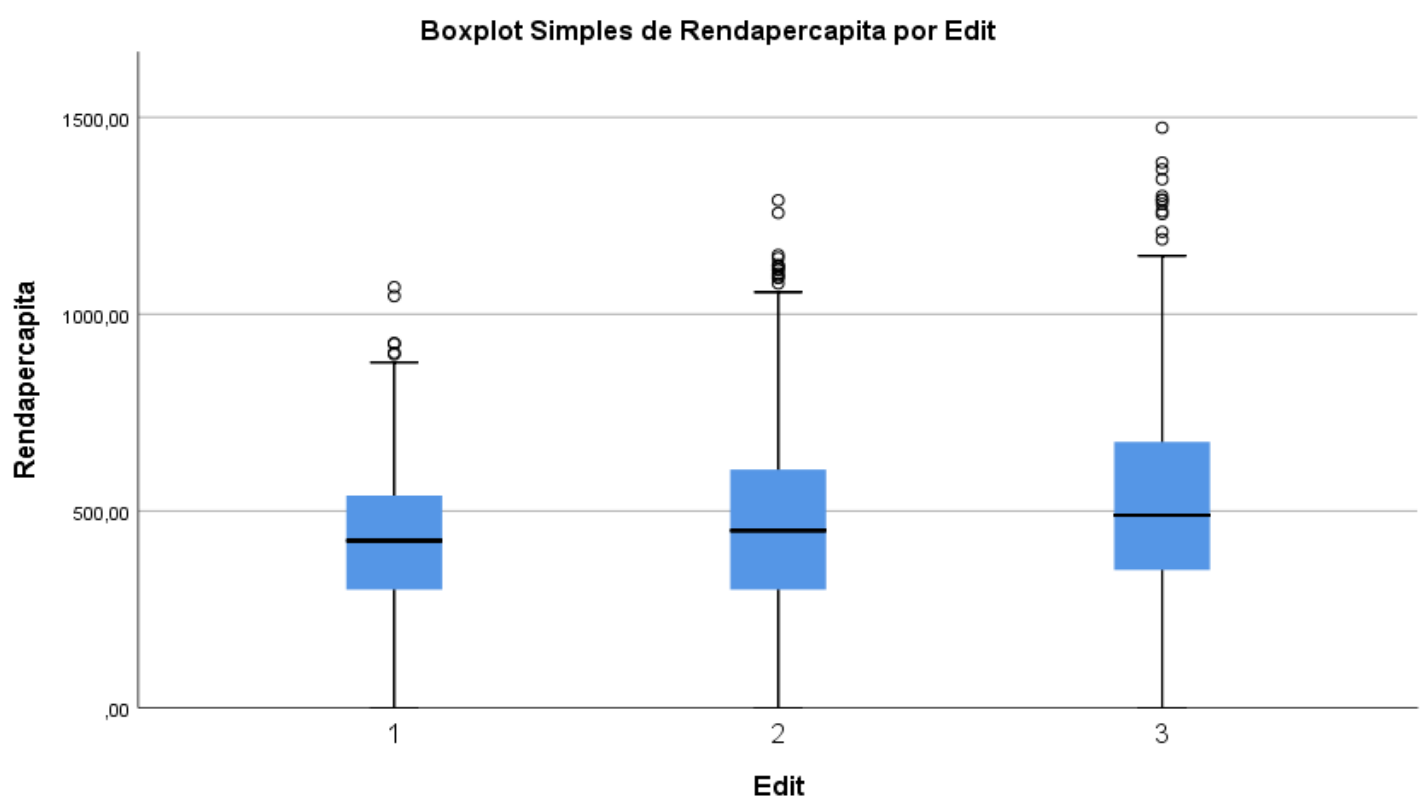

Fonte UTFPR/ASSAE 2017/2018/2019 Sistematização própria.

Apesar das mudanças, tanto em relação à pontuação obtida quanto ao aumento da renda per capita média, cabe ressaltar, tendo em vista que os indicadores e a pontuação atribuída sofreram alterações, que a mudança não indica necessariamente uma diminuição da vulnerabilidade dos acadêmicos. Ademais, é essencial destacar que, ainda que o estudo de medidas repetidas e os dados sobre a variável renda indiquem um aumento tanto na renda bruta quanto na renda per capita das famílias, a renda obtida com o auxílio estudantil é comprometida com as necessidades do acadêmico (com alimentação, moradia, transporte, material didáticopedagógico), não significando, necessária e diretamente, uma melhoria nas condições de vida do grupo familiar.

O estudo de medidas repetidas e os dados da variável renda demonstram o aumento da renda, tanto total quanto per capita, para os estudantes que permanecem como contemplados no programa: um vigoroso argumento a favor da continuidade e, sobretudo, da permanência ininterrupta dos estudantes no programa (há que se pensar em possibilidades de renovação automática, sendo revistos apenas os casos em que, manifestadamente, houver mudança na condição das famílias). 


\section{Considerações finais}

A elaboração desse instrumental (IVA-UTFPR) perpassa questões de ordem teórica, normativa, técnica, metodológica e empírica, sobretudo, decisões de gestão. A utilização cada vez mais recorrente do índice de vulnerabilidade, em âmbito acadêmico, revela a necessidade de estudos, pesquisas e reflexões, que possam servir de embasamento e contribuir à problematização e utilização dessa ferramenta/instrumento.

No que se refere à aplicabilidade do IVA-UTFPR, a pesquisa estatística revelou que, apesar da relevância do indicador de renda per capita, esta não é a única, sendo que outros indicadores demonstram influência na composição do IVA-UTFPR, os quais são: 'reside com', 'condição de permanência na residência da família', 'situações atípicas', tendo influência na formação/composição do IVA-UTFPR. Ainda no quesito dados do indicador renda, um fator importante revelado pela pesquisa é que tanto a renda total quanto a renda per capita dos estudantes/familiares aumentou progressivamente nos anos estudados.

O estudo de causa/efeito demonstrou os indicadores com maior peso percentual tanto na composição do índice como na composição do ranking de classificação. Atualmente, o indicador de renda per capita continua sendo o indicador definidor do "peso" do índice de vulnerabilidade, contudo, não é mais o único indicador. A utilização do índice demostra, ainda, que há correlação positiva entre alguns indicadores utilizados, ou seja, há associação entre as variáveis e, quando uma é alterada, a outra também se altera.

A distribuição e peso dessas variáveis corroboram a concepção multidimensional das dificuldades vivenciadas pelos estudantes. Uma das principais descobertas desta pesquisa, corroborando a hipótese que norteou este estudo, é a comprovação estatística de que outras dimensões compõem o índice de vulnerabilidade acadêmica e o ranking de classificação geral dos estudantes, além de contribuir para identificação dos principais obstáculos à permanência acadêmica.

O estudo realizado apontou, ainda, que algumas dimensões e indicadores possuem baixa validade estatística, devendo ser observados pela sua relevância social, dadas as concepções da equipe técnica que os elegeram.

A compreensão de que o IVA-UTFPR busca evidenciar os fatores que provocam riscos de não permanência pondera a adequação de indicadores que podem ser intitulados como 'acadêmicos', considerando o percurso formativo dos estudantes, fundamental para expressar as interfaces do modo como os estudantes se vinculam à instituição e aos cursos, além de evidenciar como se entende parte do processo, considerando alguns 'dilemas pedagógicos'. 
Desse modo, é importante considerar questões como desempenho acadêmico, retenção, evasão, carga horária, participação em atividades complementares, participação em grupos de pesquisa e extensão, vínculo estabelecido com seus professores, sentimento de pertencimento ao curso e à instituição, adequação das necessidades formativas ao perfil da instituição e do estudante, processos avaliativos, metodologias didáticas, etc.

Considerando o exposto nesta pesquisa e a compreensão de que os indicadores não constituem um objetivo, mas antes uma ferramenta/instrumento, conclui-se que as etapas na sua definição e utilização requerem clareza e rigor metodológico. Conforme aponta Januzzi (2002), os indicadores devem ser coerentes com os objetivos de cada instrumento e das políticas que operacionalizam. Nesse sentido, é essencial, em face de cada indicador, se perguntar que situação, fenômeno ou fator está se retratando. Dessa forma, para utilização adequada dos indicadores, são necessárias algumas reflexões: O que será medido? Qual é a informação necessária? Quais são os valores de comparação ou referência? Como será obtida a informação? Para responder a essas perguntas, é necessário conhecer as condições ou características do fenômeno a que se refere. Esse processo de reflexão e adequação deve ser constante, acompanhando, na medida do possível, o movimento do concreto e do cotidiano.

Nessa linha argumentativa, compreendemos que o IVA-UTFPR, enquanto um instrumento de identificação e intervenção na vulnerabilidade acadêmica, pode ser melhorado a partir da fundamentação teórica e da análise estatística da sua aplicabilidade, bem como revisão constante. Os dados estatísticos, expostos nesta pesquisa, demostram a necessidade de ajustes em alguns indicadores, pois não apresentam relevância estatística, necessitando adequar as concepções e escolhas da equipe técnica com os parâmetros para identificação de incidência de maior ou menor grau, nas situações de vulnerabilidade acadêmica, o que indica maior influência à possibilidade de evasão.

Finalmente, compreendemos que a pesquisa realizada permite-nos identificar o IVAUTFPR como critério de classificação mais adequado para o programa de AE, se comparado ao critério renda per capita, pois contribui para alcançar os estudantes com diversas dificuldades, as quais incidem na vulnerabilidade acadêmica e influenciam, em maior ou menor medida, na permanência dos estudantes, objetivo principal da assistência estudantil.

\section{Referências}

BRASIL. Decreto n ${ }^{\circ}$ 7234, de 19 de julho de 2010. Dispõe sobre o Programa Nacional de Assistência Estudantil - PNAES. Brasília-DF, 2010. Disponível em: 
http://www.planalto.gov.br/ccivil_03/_Ato2007-2010/2010/Decreto/D7234.htm. Acesso em: 15 ago. 2018.

CARVALHO, M. A educação superior no brasil: o retorno privado e as restrições ao ingresso. Revista Sinais Sociais, Rio de Janeiro, v. 5, n. 15., p. 82-109, 2011. Disponível em: http://www.sesc.com.br/portal/publicacoes/sesc/revistas/sinaissociais/n15/n15. Acesso em: 15 set. 2019.

FONAPRACE. V Pesquisa Nacional de Perfil Socioeconômico e Cultural dos estudantes de graduação. Brasília: ANDIFES, 2019. Disponível em: http://www.andifes.org.br/wpcontent/uploads/2019/05/V-Pesquisa-Nacional-de-Perfil-Socioeconômico-e-Cultural-dos-asGraduandos-as-das-IFES-2018.pdf. Acesso em: 19 dez. 2019.

JANUZZI, P. M. Considerações sobre o uso, mau uso e abuso dos indicadores sociais na formulação e avaliação de políticas públicas municipais. Revista de Administração Pública, Rio de Janeiro, v. 36, n. 1, p. 51-72, 2002. Disponível em:

http://bibliotecadigital.fgv.br/ojs/index.php/rap/article/view/6427. Acesso em: 13 ago. 2019.

MAURIEL, Ana. Paula. O. Combate à pobreza e desenvolvimento humano: impasses teóricos na construção da política social na atualidade. 2008. Tese (Doutorado em Ciências Sociais) - Instituto de Filosofia Ciências Humanas, Universidade Estadual de Campinas, Campinas, 2008.

ROQUE, Antônio. Estatística II. Aula 13. Curitiba, 2009. Disponível em:

http://sisne.org/Disciplinas/Grad/ProbEstat2/aula13.pdf. Acesso em: 01 abr. 2019. 